schaftliche, rechtliche, naturwissenschaftliche und sozialwissenschaftliche Behandlung des ökologischen Problemfeldes. Dafür werden z.B. aus dem naturwissenschaftlichen Bereich Veranstaltungen angeboten zu den Themen ,Wasser - Stoff zum Leben - Lebensraum Gewässer" und „Technische Verfahren zum Schutz der Umwelt". 4. Aus diesen fünf Fächern sollen die Studierenden ein Vertiefungsfach wählen, in dem sie über die vier SWS hinaus weitere acht $\mathrm{zu}$ absolvieren und nicht nur eine einfache, sondern eine doppelte Prüfungsleistung zu erbringen haben (Vorund Hauptleistung). Beispielsweise sollen für die naturwissenschaftliche Vertiefung zwei zweistiundige Veranstaltungen zu den Themen ,Die Atmosphäre. Entstehung, Entwicklung, Prozesse (Wetter, Klima), Gefährdung“ sowie „Sedimente und Böden - die Registratur der Sünden - Altlasten und ihre Bewältigung“ bereitgestellt werden, ferner ein Praktikum „Umweltanalytischer Grundkurs“ und/oder ein Betriebspraktikum in der Arbeitsgruppe Ökochemie und Umweltanalytik.

5. Auf der anderen Seite dieses ökologiebezogenen Hauptstudiumteils verbleiben je vier SWS allgemeine Betriebs- und Volkswirtschaftslehre sowie zwei weitere Wahlpflichtfächer von zweimal zwölf SWS, wovon eines entweder aus der Betriebs- oder Volkswirtschaftslehre gewählt werden muß. Damit ist jenseits der ökologischen Ausbildungsinhalte ein genügend gründliches allgemeines wirtschaftswissenschaftliches Fundament für die Examensqualität gegeben.

6. Für die Konzeption des künftigen Grundstudiums ist von Belang, daß - ebenfalls ohne zusätzliche Stellen und hier in Kooperation mit dem Fachbereich Informatik - parallel auch ein Studiengang Wirtschaftsinformatik eingerichtet werden soll. Die wesentliche Idee für das Grundstudium heißt nun, daß es auf alle vier Diplomstudiengänge einfuihrend vorbereiten soll. Ökologische Lehrinhalte werden dementsprechend bereits in das Grundstudium integriert und gehören damit zum Pflichtkanon auch für alle diejenigen Studierenden, die sich für einen anderen Diplomstudiengang als den mit ökologischem Schwerpunkt entscheiden. Konkret werden vier SWS des Grundstudiums inklusive verpflichtender Prüfungsleistung dem Ökologiebezug gewidmet werden. Weil die Rechtswissenschaft im Oldenburger Grundstudium aufgrund der konkreten Fachbereichskonstruktion bereits eine sehr starke Stellung einnimmt, werden diese vier SWS pragmatischerweise von den anderen vier Fächern getragen.

\section{Der Autor}

Reinhard Pfriem ist Professor der Betriebswirtschaffslehre an der Universität in Osnabrück.

Kontakt: Reinhard Pfriem, Carl-von-OssietzkyUniversität Oldenburg, Institut BWLI, Postfach 2503,26111 Oldenburg. Tel. (0441) 798-8345 (Sekr. -8356)

Nach Absprache der betreffenden Kollegen soll während eines Zyklus eine gemeinsame Problemstellung behandelt werden (z.B. nachhaltige Entwicklung). Selbstverständlich wird darauf geachtet, daß diejenigen Studierenden, die nach dem Grundstudium Oldenburg verlassen wollen, keine Akzeptanzprobleme an anderen Universitäten bekommen.

7. Natürlich kann es nicht die Funktion der Einführung eines neuen Studienganges sein, die überfälligen didaktischen, prüfungstechnischen und berufsbildbezogenen allgemein erforderlichen Studienreformen im Sinne von Gratiseffekten gleich mitzuerledigen. Gleichwohl lehren die praktischen Erfahrungen im Bereich ökologischer Unternehmenspolitik und verwandten Feldern, daß die universitäre Ausbildung mehr als bisher üblich (leider auch in Oldenburg) die sozialen, kommunikativen und organisatorischen Kompetenzen der Studierenden stärken muß. Das erfordert seitens der Lehrenden, die jeweils existierenden Studien- und Prïfungsordnungen maximal in dieser Richtung auszunutzen bzw. verändern zu helfen. Wer sich im Unternehmen bewähren will, wird dies nicht hauptsächlich durch Klausurenschreiben schaffen...

Einen konkreten Ansatz in dieser Richtung versuchen wir mit der Festlegung, daß im gewählten ökologischen Vertiefungsfach entweder der Vor- oder der Hauptleistungsschein erworben werden soll durch eine

kommunikative Leistung (Arbeitsgruppe leiten, Tutorium, Studierendenübung von einem Semester), oder eine

organisatorische Leistung (Organisierung von Veranstaltung(en) oder Exkursion(en) oder eine

- kooperative Leistung (Auftun einer Mentorfirma, Praktikum unter bestimmten Fragestellungen mit Praktikumsbericht).

Kommunikation und Kooperation gelten natürlich auch für uns selber: Wir freuen uns natürlich über Anregungen, Kritik und Kontaktaufnahme.

Zur Notwendigkeit von gemeinsamen wissenschaftlichen Anstrengungen

\title{
Forschungskooperation für eine global nachhaltige Entwicklung
}

\section{Der Autor skizziert die Grundlage für eine gemeinsame Forschung und weitere Aktivitäten für eine global nachhaltige Entwicklung. Ein Aufruf zu einer inter- disziplinäre Forschungskooperation.}

$\mathcal{1}^{\circ}$ ber Nachhaltige Entwicklung gibt es bisher keine einheitliche Vorstellung. Dies dürfte angesichts der unterschiedlichen Ausgangsbedingungen in den verschiedenen Weltregionen und angesichts divergierender Interessen sozialer Gruppen sowie als Folge unterschiedlicher ethischer Orientierungen auch in Zukunft kaum erreichbar sein. Die Spannweite der Konzepte der nachhaltigen Entwicklung reichen vom Konzept des nachhaltigen Wirtschaftswachstums bis zu Nachhaltigkeitsvorstellungen, die jedweden Eingriff in die globalen Ökosysteme ausschließen. Die erste extreme
Position ist eine Umschreibung der in eine Krise geratenen Modernisierungsstrategie und die letzte Extreme stellt das Existenzrecht selbst der gegenwärtigen Weltbevölkerung von rund fünf Milliarden radikal in Frage.

Anzustreben ist eine global nachhaltige Strategie, die ökonomische, ökologische, soziale, politische und kulturelle Dimensionen einschließt. Diese ganzheitliche Entwicklungsstrategie ist zweifelsohne weitreichender als die eindimensional auf ökonomisches Wachstum orientierten Modernisierungskonzepte. Gleichwohl bedeutet sie sowohl für die Wissenschaft als auch für die Praxis unvergleichbar größere 
Anstrengung und Herausforderung: Einerseits stellt eine ganzheitliche Nachhaltigkeit zunächst die globalen Gegenwartsprobleme in ein mehrdimensionales Koordinatensystem und öffnet damit auch den methodischen Zugang zu neuen Ufern. Andererseits wirft sie viele neue Fragen auf, die erst nach umfangreichen Forschungsanstrengungen zu beantworten sind. Zwar hat die Forschung über die Nachhaltigkeit vielerorts bereits begonnen, sie steckt dennoch in den Anfängen. Die Komplexität der globalen Zusammenhänge und die Dringlichkeit globaler Reformen zwingen zu einer interdisziplinären und intensiven Forschungskooperation. Eine Verständigung über die intersektoralen, interregionalen (also horizontalen) sowie intrasektoralen und intraregionalen (also vertikalen) Interdependenzen globaler Nachhaltigkeit und die sich daraus ergebenden offenen Fragen dürfte dabei einen wichtigen Schritt darstellen. Der folgende Beitrag stellt einen Versuch in diesem Sinne dar. Er soll dazu dienen, eine Grundlage für gemeinsame Forschung und darüber hinausgehende wissenschaftliche Aktivitäten zu skizzieren, und er soll helfen, gemeinsame Projekte zu definieren. Hierzu zählen Tagungen, Publikationen, interdisziplinäre Forschungsprojekte und die Einrichtung von Graduiertenkollegs. Der Verfasser ruft alle interessierten Kolleginnen und Kollegen zu einer Kooperation auf.

\section{Offene Fragen für die weifere Forschung}

Bei den unten dargestellten Forschungsschwerpunkten sollen folgende sieben Nachhaltigkeitsregeln als Orientierung dienen:

(1) starke ökologische Nachhaltigkeit,

(2) soziale Nachhaltigkeit,

(3) politische Nachhaltigkeit,

(4) nachhaltige Verteilungsgerechtigkeit,

(5) nachhaltige Arbeitsteilung und nachhaltiger Handel,

(6) Vermeidung von Kostenexternalisierung und

(7) Bewahrung der kulturellen Vielfalt.

Die Forschungsschwerpunkte beinhalten zahlreiche Einzelfragen, die jeweils umfangreiche Forschungsarbeiten voraussetzen. Die Aufzählung dient der Vollständigkeit der Systematik und unterstellt in keiner Weise, daß die Fragen alle ganz offen sind und daß die Forschung in allen Fällen bei Null anfangen müßte. Zum einen finden zu Einzelfragen der globalen Nachhaltigkeit bereits intensive Forschungsanstrengungen statt. Und zum anderen liegen wertvolle sozialwissenschaftliche Forschungsergebnisse aus den vergangenen Jahrzehnten vor, die allerdings im Lichte von Zielen und Leitprinzipien der global nachhaltigen Entwicklung neu interpretiert werden müßten. Die Formulierung der Fragen in der folgenden Skizze ergibt sich durch die Gegenüberstellung von normativen Zielen und Leitprinzipien nachhaltiger Entwicklung und der historischen und gegenwärtigen Realität von national und international nichtnachhaltigen Wirtschafts- und Sozialstrukturen.

\section{Globale Nachhaltigkeit und Geschichte}

Welches sind die historischen Ursachen von nichtnachhaltigen Strukturen? Waren die vorindustriellen Gesellschaften nachhaltig? Wenn nichtnachhaltige Strukturen in direktem Zusammenhang mit Industrialisierung und Modernisierung stehen, so stellt sich die Frage, welche der drei Möglichkeiten dafür die Hauptverantwortung tragen: (a) die technologische Revolution an sich, (b) die historisch-politischen Rahmenbedingungen, die zügellose Eingriffe in die Ökosysteme nicht verhinderten oder (c) eine Kombination von (a) und (b)? Wären historisch nachhaltige Modernisierungsprozesse denkbar gewesen, und wenn ja, wie und unter welchen Rahmenbedingungen? Welche Aufschlüsse kann die Beschäftigung mit der Geschichte für die Gegenwart und für die nachhaltige Entwicklung in der Zukunft liefern?

\section{Nachhaltigkeit, Marktwirtschaft und Bedürfnisstruktur}

Setzt die kapitalistische Akkumulation (Arbeitsprozeß, Verwertungs- und Verteilungsprozeß) das Wachstum voraus? Wenn ja, welche Art von Wachstum: Wachstum der materiellen Produktion, oder ist es auch denkbar, daß das Modell eine Reduktion der Stoffströme, d.h. auch der mengenmäßigen Warenströme zuläßt? Worauf beruht der faktische Schwerpunkt der materiellen Produktion mit immensem Umweltverbrauch im historischen Kapitalismus:

(a) auf der eigentïmlichen Dynamik der kapitalistischen Akkumulation,

(b) auf den historischen Bedürfnisstrukturen der Menschen im Modernisierungsprozeß oder (c) auf einem Wechselspiel beider Faktoren? Wenn es im Kapitalismus einen Wachstumszwang gibt, welches sind dann die Haupttriebkräfte dieses Wachstums? Besteht ein Zusammenhang zwi- schen Zins und Wirtschaftswachstum, und ist das internationale Kredit- und Finanzsystem im Sinne nachhaltiger Entwicklung reformierbar? Können die Triebkrafte des internationalen Wettbewerbs und der Globalisierung, die in der Praxis nationale und regionale Reformen schwer beeinträchtigen, eingedämmt werden, ist ein global gebändigter Kapitalismus überhaupt vorstellbar und wenn ja, wie müssen die Rahmenbedingungen beschaffen sein?

\section{Nachhaltige Entwicklung und Beschäftigung}

Grundsätzlich sind folgende zwei Alternativen der Einkommensentwicklung und Einkommensverteilung in den Industrieländern des Nordens denkbar:

- Szenario (A): Beschäftigung und Volkseinkommen bleiben unverändert, es fỉndet jedoch eine Verlagerung der Beschäftigung von der materiellen zur immateriellen Produktion statt.

Welche Auswirkungen hat die Reduktion von Stoffströmen in den Industrieländern auf die Beschäftigung: Können anstelle der verlorengegangenen Arbeitsplätze im Bereich der materiellen Güterproduktion entsprechende Arbeitsplätze im Dienstleistungssektor geschaffen werden, und kann dabei eine Vollbeschäftigung erreicht werden? Welche Auswirkungen werden beispielsweise $\mathrm{CO}_{2}$-Reduktionsstrategien auf die Automobilindustrie haben? Welche Alternativen sind hier denkbar und nachhaltig:

(a) Produktion von Energiesparautos,

(b) Reduzierung der Automobilproduktion bei gleichzeitiger Energieverbrauchssenkung? Was folgt daraus für die Beschäftigungs- und Einkommensentwicklung in den Automobilproduktionsländern? Wie muß dann der Dienstleistungssektor beschaffen sein, damit Nachhaltigkeit im umfassenden Sinne gewahrt bleibt? Welche Folgen hat die Verlagerung von der materiellen zur immateriellen Produktion für das Steuer- und Finanzsystem und wie soll die Umschichtung der Beschäftigungs- und Bedürfnisstrukturen finanziert werden? Entstehen bei diesem Umschichtungsprozeß Finanzierungslücken, sind diese Lücken unüberwindbar oder wären sie durch Umverteilung prinzipiell zu überwinden? Welche Folgen haben die notwendigen Aufwendungen für den Umweltschutz (Beseitigung der Altlasten und Vermeidung von neuen Schäden) und für den Einkommens- bzw. Technologietransfer in den Süden auf das Modell? 
Szenario (B) Beschäftigung und Volkseinkommen sinken durch Arbeitszeitverkürzung und es findet gleichzeitig eine Verlagerung von der materiellen Güterproduktion zum Dienstleistungssektor statt.

Ist diese Alternative überhaupt von Bedeutung, wenn die Alternative (A) funktioniert? Ist diese nach zwei Seiten gerichtete Reformvariante (Strukturwandel durch Erweiterung des Dienstleistungssektors einerseits und Arbeitszeitreduzierung andererseits) systemkonform? Welche Arbeitszeitmodelle, welche Gesellschaftsmodelle durch steigende Freizeit sind dann nachhaltig? Wie soll die Freizeit bei drastischer Arbeitszeitverkürzung gestaltet werden? Wie findet der soziale Ausgleich bei unteren Einkommen statt, und wie soll dann der Umweltschutz sowie der Nord-Süd-Ausgleich finanziert werden? Oder könnte eine drastische Arbeitszeitverkürzung und ein intersektoraler Strukturwandel im Norden per se wegen sinkendem Umweltverbrauch als ein wichtiger Beitrag zur Überwindung des Nord-Süd-Gefälles gesehen werden?

\section{Nachhaltigkeit und Entwicklung in den Ländern des Südens}

Welches sind die Folgen der Reduktion des Naturverbrauchs für die Länder des Südens? Werden dadurch zwangsläufig die Exporteinnahmen dieser Länder sinken und diese Länder dadurch in neue Krisen gedrängt, oder ist es grundsätzlich möglich, dramatische und kontraproduktive Einbrïche und Entwicklungsblockaden für den Süden in diesem Prozeß zu verhindern? Ist es denkbar, globale Rahmenbedingungen herzustellen, damit erschöpfbare Ressourcen statt zu Dumpingpreisen zu Knappheitspreisen verkauft werden? Wenn ja, welches sind dann die Rahmenbedingungen und welche Politik muß dann verfolgt werden?

Müssen Länder des Südens den geschichtlichen Werdegang des Industrialisierungsprozesses der Industrieländer nach denselben organisatorischen und technologischen Mustern nachholen oder kann dieser Weg ökologisch und sozial abgekürzt werden? Wenn ja, welche Rahmenbedingungen, Instrumente und Prioritäten sind hierfür notwendig?

\section{Verhältnis Nachhaltigkeit und politisches System}

Wie soll bei einem Höchstmaß von demokratischpartizipatorischen Strukturen ein innergesell-

\section{Der Autor}

Mohssen Massaral ist Professor für Politikwissenschaft an der Universität Osnabrück mit dem Schwerpunkt internationale Wirtschaftsbeziehungen. Dieser Beitrag ist die gekürzłe Fassung eines Papiers des Autors, in dem er die Notwendigkeit einer solchen Forschungskooperation und gemeinsamer wissenschaftlicher Aktivitäten begründet. Die ungekürtze Fassung sowie weitere Informationen sind direkı beim Autor erhältlich.

Kontakt: Universität Osnahrück, Fachbereich Sozialwissenschaften, 49069 Osnabrück, Tel. (0541) 969-4156, Fax-4600

schaftlicher Interessensausgleich stattinden, wie wird ein politischer Konsens hergestellt und welche Bedeutung kommt sozialen Bewegungen und Nichtregierungsorganisationen zu? Wie soll sich das Verhältnis der Nachhaltigkeit zur individuellen Freiheit gestalten? Setzt Nachhaltigkeit der Freiheit des Konsums, der Produktion und des Eigentums, insbesondere an Naturreichtïmern, Grenzen und wie? Welches wären dann Kriterien für einen neuen universellen Freiheitsbegriff?

\section{Nachhaltigkeit, zentralistische und dezentralistische Strukturen}

Gibt es einen Zusammenhang zwischen Nachhaltigkeit und zentralistischen bzw. dezentralistischen politischen und ökonomischen Strukturen bei Warenproduktion, Handel, Bankwesen, Energieversorgung, Verwaltung, Massenmedien etc.? Wenn ja, sind zentrale oder dezentrale Strukturen oder eine Kombination von beiden nachhaltig und welches sind Kriterien einer Differenzierung? Wie müssen die lokalen, nationalen, regionalen und globalen Ebenen und Zuständigkeiten im Sinne umfassender Nachhaltigkeit miteinander gekoppelt werden? Welche Aufgaben und Entscheidungen obliegen der lokalen, der nationalen bzw. der regionalen Autonomie und welche Aufgaben sind universell und fallen in den Verantwortungsbereich der Weltgemeinschaft?

\section{Globale Nachhaltigkeit, Stever ungsinstrument Weltarbeits- teilung}

Welche Instrumente und Maßnahmen sind geeignet, in den Industrieländern und in den Ländern des Südens einen Umstrukturierungsprozeß, der zur nachhaltigen Entwicklung fuihrt, wirkungsvoll in Gang zu setzen: (a) Steuern und Abgaben, (b)
Zertifikate und Lizenzen, (c) Mengenbegrenzung oder (d) eine Kombination dieser Instrumente? Kann der Umstrukturierungsprozeß unter Beibehaltung bestehender Rechtssysteme stattfinden oder sind Verfassungsänderungen und Reform der internationalen Rechtssysteme (z.B. des Völkerrechts), der Normen und internationalen Verträge erforderlich? Welche Bedeutung kommt dabei der UNO und den UNO-Unterorganisationen zu? Tangiert die Nachhaltigkeit die Souveränität von Staaten, in welchen Zusammenhängen und welche Lösungsmöglichkeiten sind hier denkbar? Wie wird und wie soll sich der internationale Handel gestalten, in Form von Freihandel oder durch dessen Beschränkung? Ist es denkbar, daß durch eine Kombination von international anerkannten Öko- und Sozialstandards mit Freihandel den Nachhaltigkeitsprinzipien Genüge getan wird? Wie wird schließlich eine nachhaltige Weltarbeitsteilung aussehen?

\section{Nachhaltigkeit und Lebensstil}

Welches sind die Determinanten des Lebensstils in den Industrieländern des Nordens und in den Ländern des Südens? Ist das Streben nach Haben und nach mehr Konsum eine anthropologische Konstante oder resultiert es eher aus dem gesellschaftlichen Evolutionsprozeß und ist es daher veränderbar? Kann ein verschwenderischer Lebensstil definiert werden und wie? Kann eine allgemeingültige Definition von nachhaltigem Lebensstil formuliert werden? Welches sind die Triebkräfte des Lebensstilwandels, z.B. Nachahmung, Aufklärung, gesellschaftliche Restriktionen, Naturkatastrophen etc.? Wie ist die Diskrepanz zwischen Wissen und Handeln zu erklären und wie kann diese überwunden werden? Wie können individuelle Ängste, die bei Menschen in Zusammenhang mit Veränderungen und Reformen anzutreffen sind, aufgefangen und wie kann verhindert werden, daß solche Ängste instrumentalisiert werden?

\section{Global nachhaltige Entwicklung und Bevölkerungswachstum}

Gibt es für eine globale Nachhaltigkeit eine Obergrenze für die Weltbevölkerung? Wenn ja, wo liegt diese Obergrenze und wie kann verhindert werden, daß sie überschritten wird? Entsprechen die gängigen bevölkerungspolitischen Maßnahmen den Nachhaltigkeitskriterien und wie muß eine nachhaltige Bevölkerungspolitik aussehen? 
(c) 20I0 Authors; licensee IÖW and oekom verlag. This is an article distributed under the terms of the Creative Commons Attribution Non-Commercial No Derivates License (http://creativecommons.org/licenses/by-nc-nd/3.o/), which permits unrestricted use, distribution, and reproduction in any medium, provided the original work is properly cited. 